\title{
Inefficient arterial hypertension control in patients with metabolic syndrome and its link to renin-angiotensin- aldosterone system polymorphisms
}

\author{
Maria M Morales-Suárez-Varela ${ }^{1,2}$, Maria L Mansego ${ }^{3,4}$, Ana M Vicedo-Cabrera ${ }^{1}$, Monica Pineda-Alonso ${ }^{5}$, \\ Aqustin Llopis-González ${ }^{1,2}$, Jose M Martin-Moreno ${ }^{6}$, Juan C Martín-Escudero ${ }^{5}$ and FJ Chaves ${ }^{3,6,7}$
}

There is evidence that uncontrolled arterial hypertension (AHT) in patients with metabolic syndrome (MS) increases cardiovascular risks. The renin-angiotensin-aldosterone system (RAAS) and its polymorphisms apparently confer a genetic risk for uncontrolled AHT. This study aims to investigate the influence of RAAS polymorphisms on AHT control in patients diagnosed with MS. This is a two-stage population-based nested case-control pilot study ( $n=1514)$. We differentiated between MS-diagnosed patients and non-MS patients (ATP-III criteria) and selected those individuals diagnosed with AHT from each group ( $n=161$ and $n=156$, respectively). Those who successfully controlled their AHT (controls) and those who did not were compared. In the MS population, the $C / G$ and $G / G$ genotypes of single-nucleotide polymorphism rs 1040288 (NR3C2) and $A / G$ and $G / G$ of rs11099680 (NR3C2) were associated with uncontrolled AHT (odds ratio (OR)=2.94 (1.34-6.47) and $O R=2.54$ (1.09-5.93), respectively). According to Akaike's information criteria, the best adjusted model included gender and age as confounding variables (adjusted $\mathrm{OR}(\mathrm{ORa})=2.91(1.31-6.46)$ and $\mathrm{ORa}=2.67(1.13-6.31)$, respectively). Regarding rs1040288, an ORa of 4.03 (1.44-11.26) was obtained for the saturated model (adjusted for gender, age, waist-to-hip ratio, body mass index, biochemical profile, renal damage, smoking habit and anti-AHT treatment). Yet, when the same analysis was performed on the non-MS population, no association was found between rs11099680 and the failure to control AHT. The results reveal a possible association between the rs 11099680 RAAS polymorphism and uncontrolled AHT in MS-diagnosed patients. rs1040288 appears to be associated with uncontrolled blood pressure regardless of MS profile.

Hypertension Research (2011) 34, 758-766; doi:10.1038/hr.2011.37; published online 7 April 2011

Keywords: arterial hypertension; metabolic syndrome; renin-angiotensin-aldosterone system

\section{INTRODUCTION}

Metabolic syndrome (MS) is recognized as a cluster of cardiometabolic risk factors associated with high cardiovascular risk, including glucose intolerance, dyslipidemia, arterial hypertension (AHT) and obesity. ${ }^{1,2}$ All of these pathologies have become major public health concerns because their components are rapidly emerging as twentyfirst century epidemics. ${ }^{3,4}$ Most authorities suggest an aggressive approach toward all the individual components of MS. ${ }^{5}$ The objective of the established therapeutic methodology to control this syndrome consists of reducing high cardiovascular risks or renal events and attempting to avoid the development of type 2 diabetes mellitus or $\mathrm{AHT}^{6}{ }^{6}$ as individuals with MS have a higher than average likelihood of developing these conditions.

Controlling blood pressure (BP) is important given that MS considerably increases cardiovascular risk and renal events. ${ }^{6}$ AHT associated with MS has pathophysiological characteristics that pose clinical challenges and provide opportunities for successful therapeutic intervention. ${ }^{7}$ Although anti-AHT therapy is considered essential in AHT management, specific BP treatment goals have not yet been introduced in this high-risk population. Despite there being no clear consensus on the most effective anti-AHT treatment, it is agreed that angiotensin-converting enzyme (ACE) inhibitors or angiotensin receptor antagonists are the best first-line therapies for MS patients. ${ }^{5}$ In fact, there is evidence that stimulating the renin-angiotensinaldosterone system (RAAS) is a factor commonly associated with obesity and insulin resistance, and this may have a key role in MS patients' high BP. ${ }^{6}$ Certain patient populations show a high prevalence for AHT, including the elderly and individuals with obesity, MS, diabetes or chronic kidney disease. Many of these patients exhibit dysregulation of the RAAS, which is relevant because this system has

${ }^{1}$ Department of Preventive Medicine, Unit of Public Health and Environmental Care, University of Valencia, Valencia, Spain; ${ }^{2} \mathrm{CIBER}$ Epidemiology and Public Health, Spain; ${ }^{3}$ Genotyping and Genetic Diagnosis Unit, Hospital Clinico Research Foundation and INCLIVA, University of Valencia, Valencia, Spain; ${ }^{4}$ CIBER Physiopathology of Obesity and Nutrition, Spain; ${ }^{5}$ Internal Medicine Department, Rio Hortega University Hospital, Valladolid, Spain; ${ }^{6}$ Department of Preventive Medicine and Health Care Quality Unit, Medical School and Clinical Hospital, University of Valencia, Valencia, Spain and ${ }^{7}$ CIBER Diabetes and Associated Metabolic Diseases, Spain

Correspondence: Dr MM Morales-Suárez-Varela, Unit of Public Health and Environmental Care, Department of Preventive Medicine, University of Valencia, Avda. Vicente Andrés Estellés s/n, 46100 Burjassot, Valencia, Spain.

E-mail: maria.m.morales@uv.es

Received 5 July 2010; revised 10 January 2011; accepted 26 January 2011; published online 7 April 2011 
key roles in the pathogenesis of AHT, cardiovascular disease and renal dysfunction. ${ }^{7}$ It is also a central regulator of cardiovascular and renal homeostasis, and its components are involved in the control of BP, body fluid homeostasis and several other cardiovascular functions. The RAAS is an endocrine system in which angiotensin II is synthesized and transported to peripheral tissues by systemic circulation, while its actions in target cells are mediated through the angiotensin type- 1 and type- 2 receptors. ${ }^{8}$ Among other functions, glomerulosa cells of the adrenal cortex secrete aldosterone when stimulated by angiotensin II via the angiotensin type-1 receptor, and aldosterone interacts with the mineralocorticoid receptor, which triggers body salt/fluid homeostasis and BP control maintenance measures. ${ }^{9}$

However, it is difficult to control BP effectively, especially in patients with other comorbidities, such as those represented by MS. Despite the close association between components of MS and high BP, it is difficult to identify their individual contributions to increased BP because they all interact with other MS components, mechanisms inducing AHT and individual genetic backgrounds. It is, therefore, a challenge to understand the exact underlying causes and consequences. The RAAS is an excellent candidate to investigate the genetic factors that control AHT in MS patients in relation to gene-environment interactions and their influence on the patients' health status. ${ }^{10-12}$ For example, Milionis et al. ${ }^{5}$ observed a possible association of the AC genotype and the $\mathrm{C}$ allele of the angiotensin II type- 1 receptor gene with a better response to structured anti-AHT treatment in MS patients.

With all this in mind, our pilot study aims to provide a method to evaluate the roles that certain RAAS polymorphisms have in effective AHT control in MS-diagnosed patients with non-MS hypertensive patients as a reference group.

\section{METHODS}

A two-stage nested case-control population-based pilot study was carried out from 1997 to 2002 in an adult sample population (15 years of age or older) in the province of Valladolid, Spain. The data of $20 \%$ of this population ( $n=35901$ ) were obtained from the 1997 Municipal Census covering our Health Area and were selected by random sampling and stratified according to age and gender. Individuals excluded from this pilot study were those over 90 years of age, non-residents in the province, patients with clear cognitive deterioration, clinically and terminally ill individuals, and subjects with a marked walking disability $(n=1514)$. This left a total sample of 34742 people, to whom an initial survey was sent by mail. In a second step, a representative subsample of the general population was selected. A second mail survey was carried out, followed by a structured face-to-face interview with closed questions $(n=1514)$.

\section{Study population}

We began this study with a sample of 1514 people aged $15-89$ years $(50.3 \%$ women, $49.7 \%$ men) (Figure 1). The sample was split into two groups according to on whether individuals had been diagnosed with MS. Those diagnosed with MS $(n=352)$ were identified by following the ATP-III criteria, ${ }^{13}$ which requires meeting at least three of the following conditions: abdominal obesity (waist circumference $>1.02 \mathrm{~m}$ in men, $>0.88 \mathrm{~m}$ in women); triglycerides $\geqslant 150 \mathrm{mg} \mathrm{dl}^{-1}\left(\geqslant 1.68 \mathrm{mmoll}^{-1}\right)$; high-density lipoprotein cholesterol $<40 \mathrm{mg} \mathrm{dl}^{-1}\left(<1.03 \mathrm{mmoll}^{-1}\right)$ in men, $<50 \mathrm{mg} \mathrm{dl}^{-1}$ $\left(1.28 \mathrm{mmoll}^{-1}\right)$ in women; BP $\geqslant 130 \mathrm{~mm} \mathrm{Hg}$ (systolic) and/or $85 \mathrm{~mm} \mathrm{Hg}$ (diastolic); and fasting plasma glucose $\geqslant 110 \mathrm{mg} \mathrm{dl}^{-1}$.

Next, each group was divided into two subgroups (AHT patients and nonAHT patients, according to their previous diagnoses). The hypertensive subsample was then further divided into two smaller groups: individuals with 'uncontrolled AHT' (cases) (39 MS patients; 64 non-MS patients) and individuals with 'controlled AHT' as controls (122 MS patients; 92 non-MS patients). AHT was considered uncontrolled in those patients who presented levels of systolic BP, diastolic BP or both equal to or higher than the limits established for AHT according to the World Health Organization criteria defining AHT and its different subtypes. ${ }^{14,15}$ For this classification, the levels used for each patient were the average of multiple measurements taken during their daily activities (over a 24-h period) using an ambulatory BP monitoring system during the study period. These BP means are not shown in Table 1.

\section{Study procedure}

Initially, a descriptive study was undertaken to determine whether there were statistically significant differences between the averages and percentages of the cases (uncontrolled AHT) and controls (controlled AHT) in each study subpopulation (AHT with and without MS). Then, the data were analyzed to identify possible associations between the presence of a particular polymorphism and the failure to efficiently control AHT among the MS-diagnosed patients. Finally, in the second stage, a further analysis was done to determine whether the associations with the polymorphisms previously identified

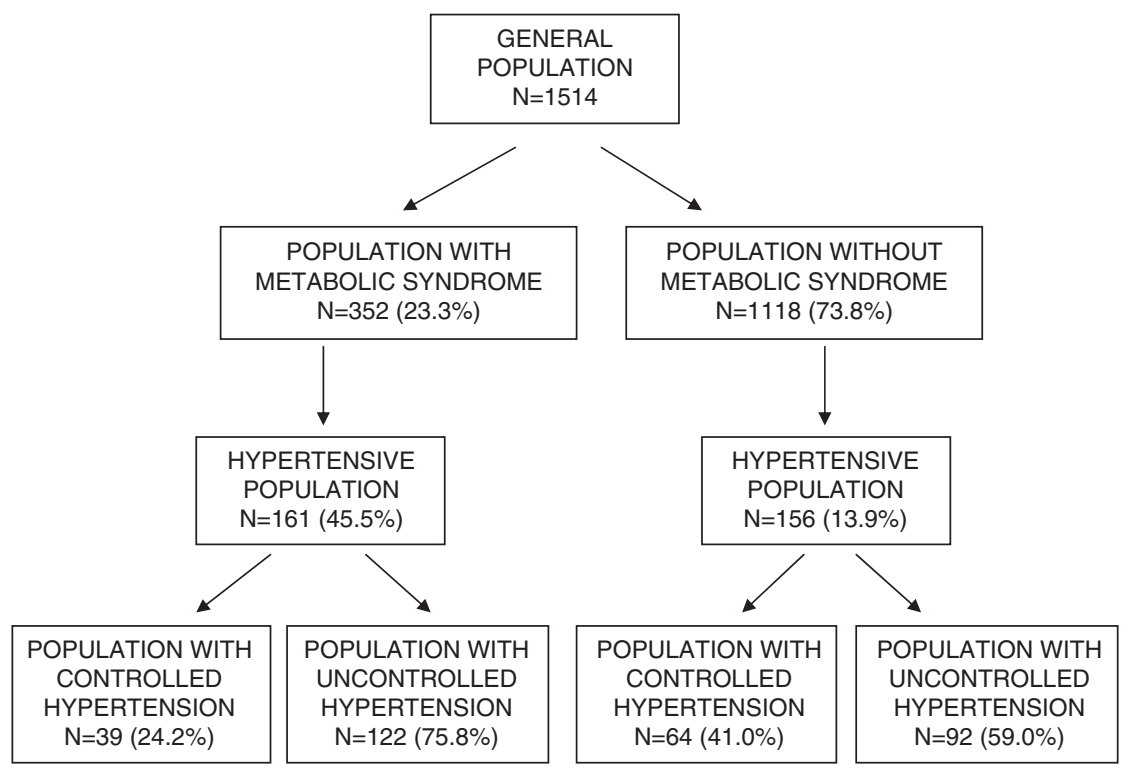

Figure 1 Sample selection criteria. 


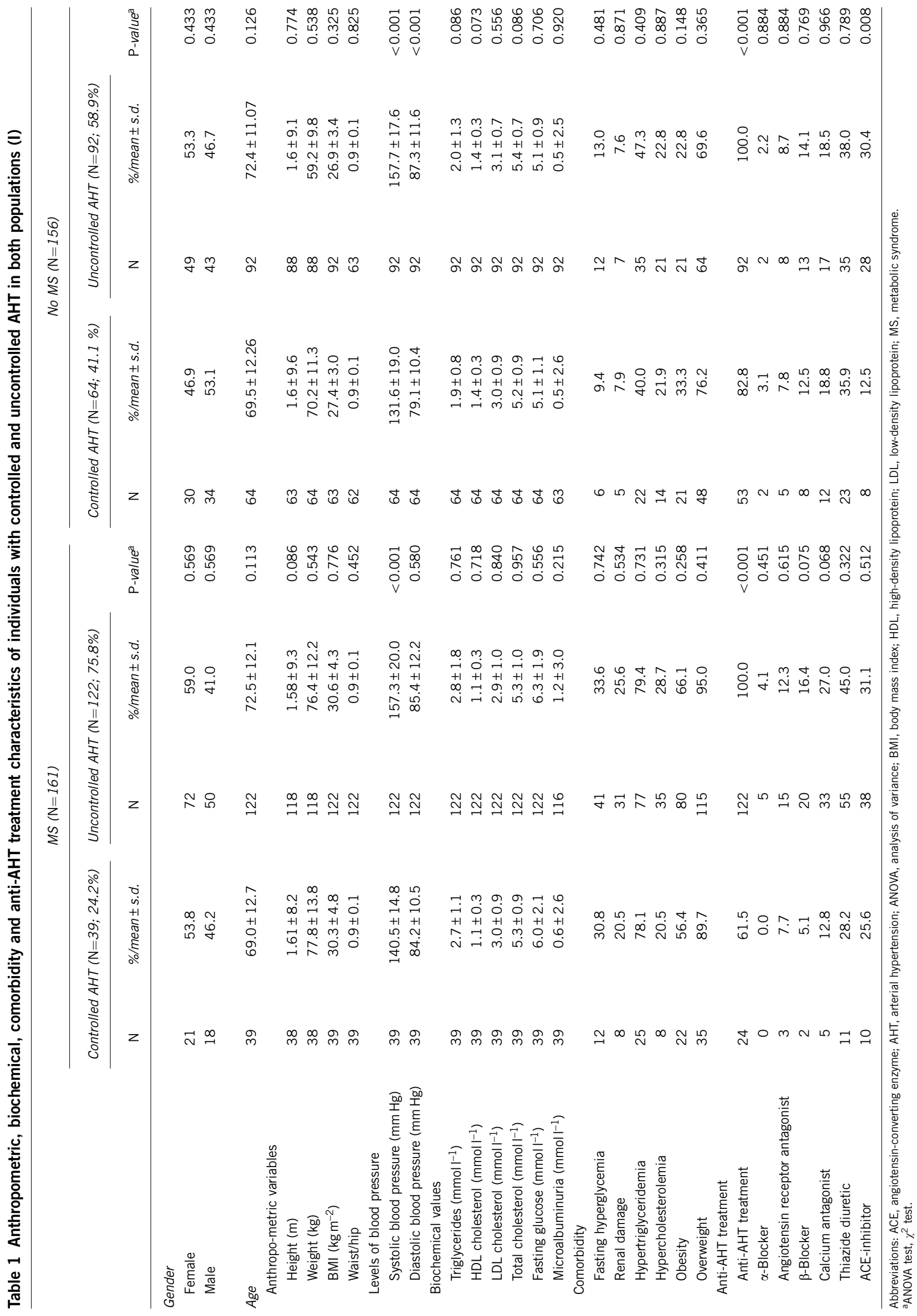


in the MS patient population were also present in the non-MS hypertensive population.

\section{Patient data}

$\mathrm{BP}$ values were measured during a doctor's visit according to the Joint National Committee's recommendations: after at least $5 \mathrm{~min}$ of supine rest, the average measurement was taken of two readings carried out 5 min apart; if the first two differed by $>5 \mathrm{~mm} \mathrm{Hg}$, more readings were taken. ${ }^{16}$ Measurements were taken with the automatic monitor OMRON-711 and validated ${ }^{17,18}$ according to the recommendations of the British Hypertension Society ${ }^{19}$ and the Association for the Advancement of Medical Instrumentation. ${ }^{20}$ The monitor was calibrated for this study (Table 1).

Other data obtained from the study subjects were anthropometric measurements (weight, height, body mass index (BMI), waist and hip) and biochemical parameters (fasting plasma glucose, triglycerides, high-density lipoprotein cholesterol, low-density lipoprotein cholesterol, total cholesterol and microalbuminuria). The presence of other conditions was also noted, particularly fasting hyperglycemia (glucose $>110 \mathrm{mg} \mathrm{dl}^{-1}$ or $6.11 \mathrm{mmoll}^{-1}$ ), hypertriglyceridemia (triglycerides $>150 \mathrm{mg} \mathrm{dl}^{-1}$ or $1.68 \mathrm{mmoll}^{-1}$ ), hypercholesterolemia (total cholesterol $>200 \mathrm{mg} \mathrm{dl}^{-1}$ or $4.17 \mathrm{mmoll}^{-1}$ ), obesity $\left(\mathrm{BMI}>30 \mathrm{~kg} \mathrm{~m}^{-2}\right.$ ) and overweight $\left(\mathrm{BMI}=25-29 \mathrm{~kg} \mathrm{~m}^{-2}\right) \cdot{ }^{13}$ Data about anti-AHT treatment were also collected; subjects were asked to indicate the kind of therapy they were receiving ( $\alpha$-blockers, $\beta$-blockers, calcium antagonists, thiazide diuretic, ACE inhibitors and angiotensin receptor antagonists) (Table 1).

In addition, other environmental factors were also recorded. Socioeconomic class (according to the National Classification of Occupations (NCO is its Spanish acronym, 1979 revision) and by following the Spanish-adapted version of the British Registrar General's Classification) ${ }^{21}$ and the level of education were recorded. Energy expenditure was assessed by determining the METS, an equivalent unit of energy consumption to the resting metabolic rate (3.6 $\mathrm{ml}$ of oxygen $\left.\mathrm{kg}^{-1} \mathrm{~min}^{-1}\right){ }^{22}$ Furthermore, smoking habits were evaluated by collecting data on the number of cigarettes smoked per day and the number of packets consumed per year. Alcohol intake was determined using a nutritional survey that included questions on the quantity of alcohol consumed daily. Finally, after signing an informed consent form, a blood sample was taken for DNA extraction from the cell pellet to carry out a genetic profile.

\section{Genotyping procedures}

The polymorphisms of the genes AGT (angiotensinogen), ACE, AGTR1 (type-1 angiotensin II receptor), AGTR2 (type-2 angiotensin II receptor), REN (renin), RENBP (renin-binding protein), NR3C2 (mineralocorticoid receptor), AGTRAP (type-1 angiotensin II receptor-associated protein) and CYP11B1 (steroid 11- $\beta$-hydroxylase) were selected on the basis of their known functional effects, possible effects and distribution along the gene, with preference given to those located in exons or in contiguous regions (Table 3). We selected polymorphisms with minor allele frequencies over $5 \%$.

DNA was isolated from peripheral blood cells using the Chemagic System (Chemagen), and samples were diluted to a final concentration of $100 \mathrm{ng} \mathrm{ml}^{-1}$. All the single-nucleotide polymorphisms (SNPs) were assessed using the SNPlex oligonucleotide ligation assay (Applied Biosystems, Foster City, CA, USA) following the manufacturer's recommendations.

\section{Statistical analysis}

Quantitative variables were expressed as the mean \pm s.d., while qualitative variables were expressed as the absolute $(n)$ and relative (\%) frequencies. We used a one-way analysis of variance to explore the differences between the quantitative variables, and $\chi^{2}$ exact tests were used to compare the qualitative variables. Any $P$-value of $<0.05$ was considered significant. A statistical power of $>0.85$ at the $<0.05$ probability level ( $P$-value) was obtained to evaluate the difference in the null hypothesis contrasted with the proportion of the MS uncontrolled AHT population and non-MS uncontrolled AHT population. The departure of SNPs from Hardy-Weinberg equilibrium was tested using a $\chi^{2}$ test. For the individual SNP association analyses, genotype frequencies were assessed by multivariate methods based on a logistic regression analysis and were analyzed with codominant, dominant, recessive, over-dominant and additive models. The best genetic model was selected using Akaike's information criteria. A multivariate analysis was carried out with which the crude and adjusted odds ratios (ORc and ORa, respectively) were determined for the different SNPs analyzed (Table 3), with 95\% confidence intervals (CIs). Various models were fitted using different combinations of confounding variables: gender; age (continuous variable); BMI (continuous variable); waist-to-hip ratio (continuous variable); plasma levels of triglycerides, fasting glucose, highdensity lipoprotein cholesterol, low-density lipoprotein cholesterol and total cholesterol; and the presence or absence of diabetes mellitus and renal damage. We also controlled for smoking habits (smoker, ex-smoker and non-smoker) and for taking anti-AHT medications ( $\alpha$-blockers, $\beta$-blockers, calcium antagonists, thiazide diuretics, ACE inhibitors and angiotensin receptor antagonists). These analyses were performed using the SNPassoc R package. ${ }^{23}$ A Bonferronitype correction was also performed in this analysis. The $\mathrm{D}^{\prime}$ and $\chi^{2} P$-values for marker independence were estimated to determine the linkage disequilibrium among all the genetic markers.

\section{Analysis strategy}

First, we performed a binary logistic regression analysis to determine the association between uncontrolled AHT and the genotype of each SNP analyzed (Table 3) in the MS-diagnosed population. In those analyses in which we obtained statistically significant ORc values, we performed a multivariate logistic regression analysis using the different adjusting models by following the step-wise method and by progressively including the aforementioned confounding variables. Next, we adopted the same analysis strategy with the non-MS population to determine whether these SNPs were associated with uncontrolled AHT in the MS-diagnosed patients.

\section{RESULTS}

MS and AHT prevalence and characteristics in controlled AHT and uncontrolled AHT patients

In the study population, a global MS prevalence of $23.3 \%(n=352)$ was detected. According to the World Health Organization criteria, ${ }^{11}$ AHT prevalence was $45.7 \%(n=161)$ and $13.8 \%$ for MS patients and non-MS patients, respectively. AHT control was only achieved in $24.2 \%$ of the MS patients and in $41.0 \%$ of the non-MS patients (Figure 1).

Table 1 shows the anthropometric, biochemical, comorbidity and anti-AHT treatment data for the MS and non-MS populations by comparing controlled (controls) and uncontrolled AHT (cases). There were significant differences between cases and controls but only in systolic BP in both groups and in diastolic BP in the non-MS group, where levels were logically higher in the uncontrolled AHT group. Statistical differences relating to anti-AHT treatment were found in both populations, where the percentage of patients on anti-AHT treatment was higher in the uncontrolled AHT patients. Furthermore, the number of patients on ACE inhibitors who failed to effectively control their AHT was statistically higher in the non-MS group.

Regarding the level of comorbidity, it is worth noting that, although there were no significant differences between the controlled and uncontrolled AHT patients, we observed a higher prevalence of fasting hyperglycemia $(33.6 \%, n=41)$, renal damage $(25.6 \%, n=31)$, hypertriglyceridemia $(79.4 \%, n=77)$, hypercholesterolemia $(28.7 \%, n=35)$, obesity $(66.1 \%, n=80)$ and overweight $(95.0 \%, n=115)$ in patients with uncontrolled AHT.

Table 2 provides the average values and prevalence of the environmental factors studied in the two populations. Regarding the level of education, a higher prevalence of controlled AHT was found for the group who had completed secondary school or obtained a high school diploma, while smokers paradoxically showed a lower prevalence of uncontrolled AHT than the non-smokers in the MS population. Moreover, for the non-MS hypertensive patients, a higher prevalence of failure to control AHT was seen among those living alone. 


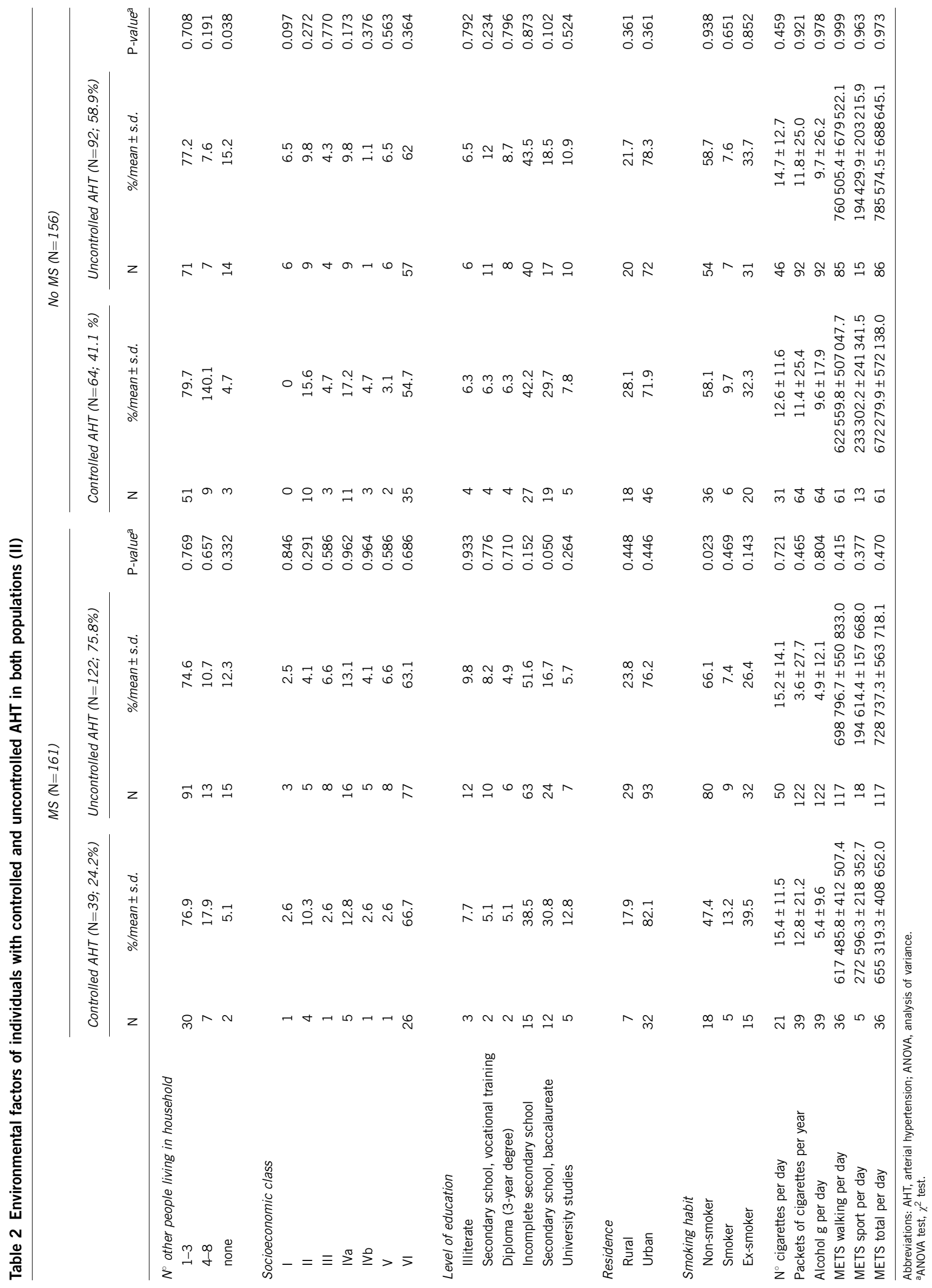


Table 3 SNPs analyzed in the sample and gene in which they are found

\begin{tabular}{|c|c|c|c|c|c|c|c|}
\hline SNP & Gene & SNP & Gene & SNP & Gene & $S N P$ & Gene \\
\hline rs4363 & ACE & rs2493132 & AGT & rs12095517 & AGTRAP & rs6535598 & NR3C2 \\
\hline Rs2229839 & ACE & rs5049 & AGT & rs4073575 & AGTRAP & rs10519952 & NR3C2 \\
\hline rs4291 & ACE & rs699 & AGT & rs6540992 & AGTRAP & rs11099680 & NR3C2 \\
\hline rs4295 & ACE & rs1492078 & AGTR1 & rs17875944 & AGTRAP & rs11724292 & NR3C2 \\
\hline rs4298 & ACE & rs275650 & AGTR1 & rs17875953 & AGTRAP & rs12498662 & NR3C2 \\
\hline rs4320 & ACE & rs2933249 & AGTR1 & rs6677313 & AGTRAP & rs1490453 & NR3C2 \\
\hline rs4331 & ACE & rs4681444 & AGTR1 & rs11571092 & REN & rs1512337 & NR3C2 \\
\hline rs4343 & ACE & rs5183 & AGTR1 & rs3215332 & REN & rs17024654 & NR3C2 \\
\hline rs4353 & ACE & rs5189 & AGTR1 & rs3795575 & REN & rs1879829 & NR3C2 \\
\hline rs4362 & ACE & rs2276736 & AGTR1 & rs6668858 & REN & rs6849903 & NR3C2 \\
\hline rs4292 & ACE & rs275649 & AGTR1 & rs5707 & REN & rs10434100 & NR3C2 \\
\hline rs4311 & ACE & rs275651 & AGTR1 & rs6682082 & REN & rs13116332 & NR3C2 \\
\hline rs4359 & ACE & rs5182 & AGTR1 & rs3730103 & REN & rs13117325 & NR3C2 \\
\hline rs4459610 & ACE & rs5186 & AGTR1 & rs11571074 & REN & rs1403142 & NR3C2 \\
\hline rs4975 & ACE & rs909383 & AGTR1 & rs11571080 & REN & rs1996025 & NR3C2 \\
\hline rs5046 & AGT & rs12695925 & AGTR1 & rs11571082 & REN & rs2070951 & NR3C2 \\
\hline rs11122576 & AGT & rs12721241 & AGTR1 & rs2269371 & RENBP & rs2137335 & NR3C2 \\
\hline rs11122580 & AGT & rs1403543 & AGTR2 & rs3027869 & RENBP & rs2248038 & NR3C2 \\
\hline rs11568020 & AGT & rs17237750 & AGTR2 & rs5945377 & RENBP & rs5529 & NR3C2 \\
\hline rs2071404 & AGT & rs17237757 & AGTR2 & rs10032250 & NR3C2 & rs7668847 & NR3C2 \\
\hline rs2071405 & AGT & rs17237792 & AGTR2 & rs1040288 & NR3C2 & rs4535 & CYP11B1 \\
\hline rs4762 & AGT & rs17231429 & AGTR2 & rs2272089 & NR3C2 & rs5299 & CYP11B1 \\
\hline rs1926723 & AGT & rs17231436 & AGTR2 & rs2871 & NR3C2 & & \\
\hline rs2071406 & AGT & rs17875979 & AGTRAP & rs5522 & NR3C2 & & \\
\hline
\end{tabular}

Abbreviations: ACE, angiotensin-converting enzyme; AGT, angiotensinogen; AGTR1, type-1 angiotensin II receptor; AGTR2, type-2 angiotensin II receptor; AGTRAP, type-1 angiotensin II receptorassociated protein; CYP11B1, steroid 11-beta-hydroxylase; NR3C2, mineralocorticoid receptor (MR); REN, renin; RENBP, renin-binding protein; SNP, single-nucleotide polymorphism.

One notable finding was that our study patients with uncontrolled AHT were undergoing anti-AHT therapy. In addition, most individuals under treatment were receiving combined anti-AHT treatments $(n=99,61.5 \%)$, particularly controlled AHT patients $(n=29,74.4 \%)$ as opposed to uncontrolled AHT patients $(n=70,57.4 \%)$ in the MS population. In contrast, lower percentages of both groups in the nonMS population were on combined anti-AHT treatment (40.6 and $32.6 \%$ ). These combinations consist of taking two or more types of drugs from the anti-AHT family, always according to the BP criteria and to the 2007 Arterial Hypertension Management Guidelines. ${ }^{15}$ No statistical differences were found for the family of anti-AHT drugs consumed in the two groups, except for the use of ACE inhibitors in the non-MS population in which the rate of use was higher among those with uncontrolled AHT.

Distribution of analyzed SNPs and single-SNP association analysis Table 3 summarizes the different SNPs analyzed in the MS population and the genes where they are located. The genotype distributions were in Hardy-Weinberg equilibrium for both the cases and controls, except for rs3795575, rs275649, rs1403543, rs17231429 and rs5945377.

When studying the association of the genotypic distribution of the analyzed SNPs with uncontrolled AHT in the MS population, statistically significant results were obtained for rs1040288 and rs11099680 (Table 4), both of which are from the NR3C2 gene (which codes for the aldosterone receptor).

Using the dominant model, the rs1040288 C/G and G/G genotypes obtained an ORc of 2.94 (95\% CI: 1.34-6.47) for uncontrolled AHT. The ORa value remained constant after performing the different adjusting models (from ORa1 of 2.91 (95\% CI: 1.31-6.47) to ORa5 of 2.85 (95\% CI: 1.17-6.98)). When we included the AHT-treatment variable in the last model, we obtained an ORa6 value of 4.03 (95\% CI: 1.44-11.26), which was greater than the others. However, according to Akaike's information criteria, the best adjusted model included age and gender (ORal $=2.91$ (95\% CI: 1.31-6.47)).

Regarding SNP rs11099680, an ORc of 2.54 was obtained (95\% CI: 1.09-5.93) for genotypes A/G and G/G (dominant model). As in the previous case, the ORa values remained constant and significant. The higher ORa value was obtained for ORa5, which was 2.98 (95\% CI: 1.14-7.76). Yet according to Akaike's information criteria, the best adjusted model with the best fit was the first one, which included gender and age, with an ORal value of 2.67 (95\% CI: 1.31-6.31). However, in this case, an increase in the ORa6 value was not observed (2.88 (95\% CI: 1.01-8.19).

Subsequently, the same statistical analysis was applied to the nonMS population in which only the SNPs rs1040288 and rs11099680 were analyzed (Table 4). The distributions of their genotypes were in Hardy-Weinberg equilibrium. Accordingly, for rs1040288 and following the recessive inheritance model, a significant ORc of 2.83 (95\% CI: 1.22-6.56) was obtained for $\mathrm{C} / \mathrm{G}+\mathrm{G} / \mathrm{G}$. This remained significant, with a similar OR in all the adjusting models, except the saturated one, where the ORa6 value considerably increased (3.42 (95\% CI: $1.22-$ 9.58)). This last adjusting model was the best according to Akaike's information criteria. For SNP rs11099680, no significant OR values were obtained in the crude analysis or in any of the tested models.

After performing the Bonferroni correction, however, no significant $P$-value was obtained in any of the fitted models.

\section{DISCUSSION}

The aim of this pilot study was to explore whether the genetic variants of the RAAS components are associated with the difficulty in 


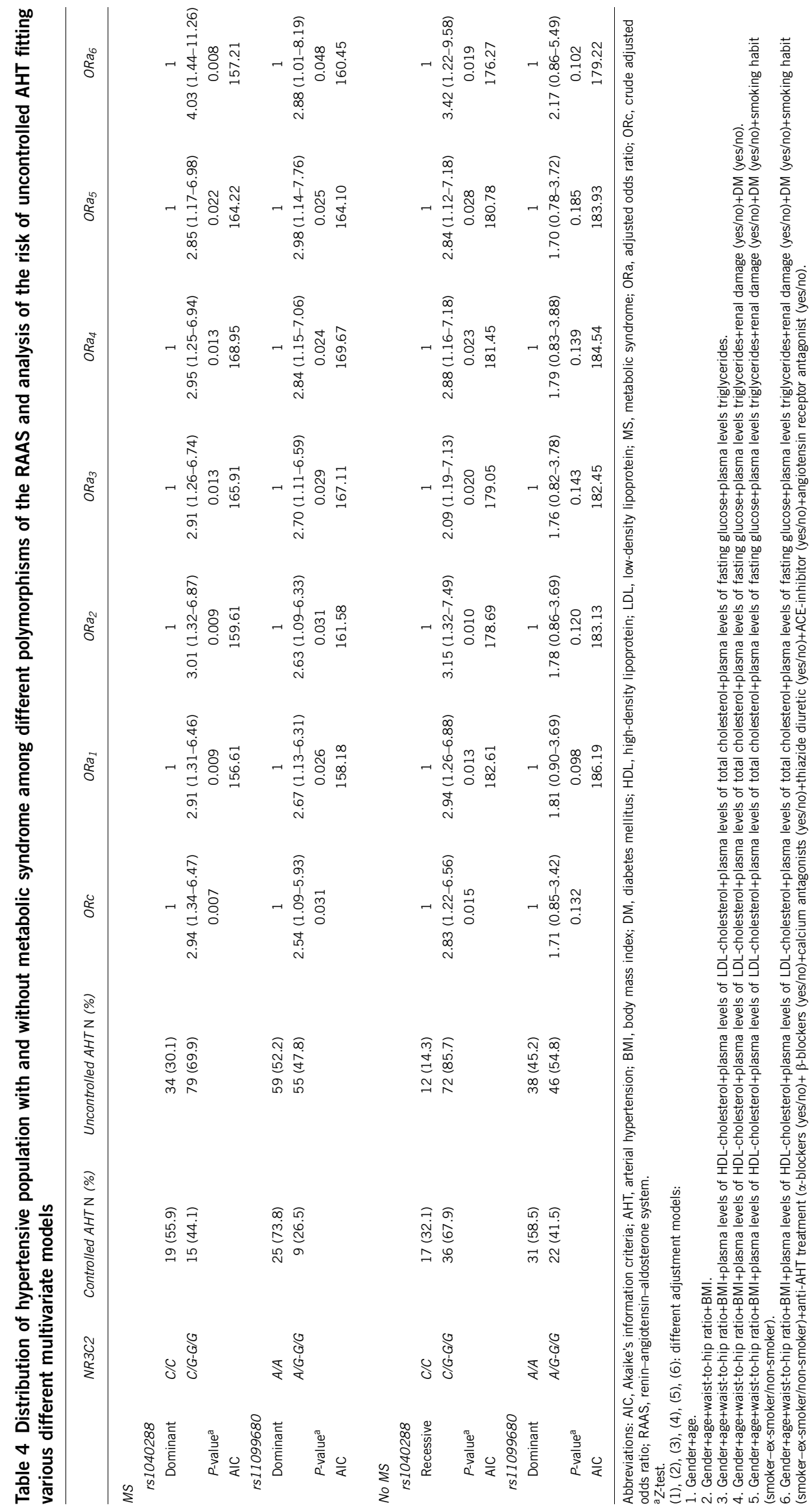


controlling BP in MS-diagnosed patients and to replicate this design in future larger studies. To date, few epidemiological studies analyzing SNPs in connection with AHT control in this population have been published, and these few have only considered a small number of SNPs. ${ }^{24}$ Our study incorporates new elements and takes another step toward improving health-care quality by analyzing the possible association of a large number of SNPs that may influence the success or failure of AHT control in MS-diagnosed patients. Indeed, our results show a possible association between the presence of the $\mathrm{G}$ allele for SNPs rs1040288 and rs11099680 (NR3C2) and the failure to control AHT in these patients. Moreover, in relation to the latter SNP, as no association was found when studying the non-MS population, it seems that the presence of this SNP could be specifically associated with the pathophysiological characteristics of MS patients, which complicates the process of controlling their BP.

In the sample population, a MS prevalence of $23.25 \%$ was seen, which is similar to that obtained during the same period in two other studies. $^{25,26}$ The sample is highly representative, so the results observed correspond to a real-life situation and not to the special trial conditions. Additionally, the fact that a third of the MS population presented controlled AHT indicates the magnitude of the influence of this metabolic alteration on BP control. Furthermore, this is supported by the fact that failure to control AHT was less prevalent in the non-MS patient group. In accordance with the presence of MS, the hypertensive profile apparently acquires certain pathophysiological characteristics that make it difficult to control BP and that should be considered when establishing both pharmacological and non-pharmacological treatments to help improve patients' health and prognosis.

Regarding the characteristics of both patient populations (MS and non-MS), no significant differences were observed between cases and controls for the anthropometric and biochemical variables (except for $\mathrm{BP})$, which illustrates the complexity of the pathological profile. Moreover, there was no clear pattern of individual characteristics that made some patients more prone to uncontrolled AHT. In fact, it seems that the factors influencing the failure to control AHT may not be anthropometric or biochemical variables but rather the patient's genetic profile. This fact has been corroborated by several studies that indicate the heritability of the factors determining the presence of MS and the development of AHT in MS patients that show that some physiological variables are as important as environmental factors, or even more so. Although the potential contribution of genetic and environmental factors to MS components has received considerable attention, current heritability estimates vary considerably. ${ }^{1}$

Similarly, in our analysis of anti-AHT treatment, the fact that no significant differences were found in the consumption of anti-AHT drug types between cases and controls, except ACE inhibitors in the non-MS population, demonstrates how difficult it is to determine the exact and most effective treatment type to control AHT, as Redon et al..$^{6}$ also stated.

Paradoxically, we observed a higher prevalence of non-smokers with uncontrolled AHT compared with smokers in the MS population. One explanation might be the potential therapeutic inertia in the AHT treatment; specifically, it could be that doctors pay more attention to AHT control in smokers as they take into account the associated cardiovascular risk profile and expected impact on health. However, this issue should be explored in further studies. ${ }^{27,28}$

Regarding the influence of the MS-diagnosed patients' genetic profile on the difficulty of controlling AHT, as stated previously, a possible association with the RAAS was observed. SNPs rs1040288 and rs11099680, whose genotypes are possible risk factors for uncontrolled
AHT, are located in the coding gene of the aldosterone, or mineralocorticoid, receptor (NC3R2). However, rs1040288 manifested this association in both populations with the same tendency, indicating that this SNP could be associated with the failure to control AHT irrespective of whether individuals have MS. Despite the two SNPs located in the same gene, it is not surprising that the results they produce differ, given that the presence of a single SNP can trigger macromolecular changes that can modify the carrier's physiology.

There is evidence that MS is a state of metabolic and vascular dysregulation that is associated with activation of the RAAS. ${ }^{29}$ More specifically, aldosterone seems to be involved in the pathogenesis of MS: in the kidney, aldosterone damages glomerular visceral epithelial cells (podocytes), leading to proteinuria and glomerulosclerosis ${ }^{3}$ and promoting the development of resistant AHT in association with obesity and MS. ${ }^{2}$ Evidence is emerging that a mineralocorticoid receptor blockade is useful in treating hypertensive patients who have both MS and resistant AHT. Accordingly, our results may explain the possible association between the G allele of the SNP rs11099680 and a higher risk of uncontrolled AHT in MS individuals. It seems that they could have acquired a series of different phenotypic characteristics: it is likely that the aldosterone-mineralocorticoid receptor interaction is one of the factors that influence the lack of AHT control in MS patients.

Findings from our multivariate analyses in the MS population suggest that components of the ATP-III criteria share common influences because important variations in the OR values were not observed in the different models. However, after adjusting for all of our variables (gender, age, waist-to-hip ratio, BMI, plasma levels of high-density lipoprotein cholesterol, low-density lipoprotein cholesterol, total cholesterol, fasting glucose and triglycerides, renal damage, diabetes mellitus, smoking habits and anti-AHT treatment), the association with uncontrolled AHT increased for rs1040288 in both study populations. This fact could reveal a possible influence of antiAHT treatment on the control of AHT in the population carrying the $\mathrm{G}$ allele of this SNP. Furthermore, this increase was not observed for rs110099680. Recent pharmacogenomics advances have revealed that some gene variants of the RAAS are involved in the effectiveness of anti-AHT treatment, especially with angiotensin II receptor blockers or the ACE inhibitor response. ${ }^{12,30}$ Indeed, this result could relate to our last study, where we found a possible association of the presence of certain polymorphisms of RAAS genes with the risk of uncontrolled HT in ACE inhibitor-treated patients. ${ }^{12}$ As noted above and by Redon et al. ${ }^{6}$ the similarity between cases and controls in the consumption of anti-AHT drug types makes it difficult to prescribe treatments to control AHT.

However, it should be taken into account that numerous calculation models were performed in the present study, so the statistical significance of this association study could be a mathematical coincidence. Furthermore, after performing the Bonferroni correction, no significant $P$-value was obtained in any of the fitted models, a fact that could represent a limitation of this study. However, it is worth noting that this is a pilot study from which the conclusions could prove to be a new initial hypothesis for subsequent studies. Similarly, it is a population-based study with strict inclusion criteria for the different groups involved. Thus, the sample size was limited, which constitutes a limitation to obtaining results that offer sufficient robustness in this genetic field.

In conclusion, these results indicate a possible association between uncontrolled AHT in MS-diagnosed individuals and the presence of certain genotypes of SNP rs11099580 in the gene coding for the aldosterone receptor (NR3C2). rs1040288 may be associated with 
uncontrolled BP regardless of the MS profile. Given the small number of patients in the final part of this study, these findings need to be replicated in larger, more heterogeneous samples for the purpose of identifying practical measures that could be taken to improve BP control and, ultimately, patients' health.

\section{ACKNOWLEDGEMENTS}

This study was supported by CIBER Fisiopatología Obesidad y Nutrición (CIBEROB) and CIBER Diabetes y Enfermedades Metabolicas Asociadas (CIBERDEM), CIBEROB and CIBERDEM are initiatives of Carlos III Health Institute, Spanish Health Ministry; Proyecto INGENFRED, ref CIBER-02-08-2009 (CIBERDEM); research grants PI070497 from the Fondo de Investigaciones Sanitarias ACOMP/2009/201, GRUPOS03/101 and PROMETEO/2009/029 from the Valencian Government and grant SAF2005-02883 from the Spanish Ministry of Education and Science.

1 Elder SJ, Lichtenstein AH, Pittas AG, Roberts SB, Fuss PJ, Greenberg AS, McCrory MA, Bouchard Jr TJ, Saltzman E, Neale MC. Genetic and environmental influences on factors associated with cardiovascular disease and the metabolic syndrome. J Lipid Res 2009; 50: 1917-1926.

2 Sowers JR, Whaley-Connell A, Epstein M. Narrative review: the emerging clinical implications of the role of aldosterone in the metabolic syndrome and resistant hypertension. Ann Intern Med 2009; 150: 776-783.

3 Nagase M, Fujita T. Mineralocorticoid receptor activation in obesity hypertension. Hypertens Res 2009; 32: 649-657.

4 Makaryus AN, Akhrass P, McFarlane SI. Treatment of hypertension in metabolic syndrome: Implications of recent clinical trials. Curr Diab Rep 2009; 9: 229-237.

5 Milionis HJ, Kostapanos MS, Vakal is K, Theodorou I, Bouba I, Kalaitzidis R, Georgiou I, Elisaf MS, Siamopoulos KC. Impact of renin-angiotensin-aldosterone system genes on the treatment response of patients with hypertension and metabolic syndrome. $J$ Renin Angiotensin Aldosterone Syst 2007; 8: 181-189.

6 Redon J, Cifkova R, Laurent S, Nilsson P, Narkiewicz K, Erdine S, Mancia G, Scientific Council of the European Society of Hypertension. The metabolic syndrome in hypertension: European society of hypertension position statement. J Hypertens 2008; 26: 1891-1900.

7 Basile J. New therapeutic options in patients prone to hypertension: a focus on direct renin inhibition and aldosterone blockade. Am J Med Sci 2009; 337: 438-444.

8 Der Sarkissian S, Huentelman MJ, Stewart J, Katovich MJ, Raizada MK. ACE2: a novel therapeutic target for cardiovascular diseases. Prog Biophys Mol Biol 2006; 91: 163-198.

9 Boon WC, Coghlan JP, Curnow KM, McDougall JG. Aldosterone secretion a molecular perspective. Trends Endocrinol Metab 1997; 8: 346-354.

10 Izawa H, Yamada Y, Okada T, Tanaka M, Hirayama H, Yokota M. Prediction of genetic risk for hypertension. Hypertension 2003; 41: 1035-1040.

11 Arnett DK, Claas SA, Glasser S. Pharmacogenetics of antihypertensive treatment. Vascul Pharmacol 2006; 44: 107-118.

12 Morales-Suárez-Varela MM, Mansego ML, Martín-Escudero JC, Llopis-González A, Chaves FJ, López-Izquierdo R, Frutos-Llanes R, Vicedo-Cabrera AM. How ineffective hypertension control in subjects treated with angiotensin-converting enzyme inhibitors is related to polymorphisms in the renin-angiotensin-aldosterone system. Eur J Pharm Sci 2010; 39: 380-386.

13 Expert Panel on Detection, Evaluation, and Treatment of High Blood Cholesterol in Adults. Executive summary of the Third Report of The National Cholesterol Education Program (NCEP) expert panel on detection, evaluation, and treatment of high blood cholesterol in adults (Adult Treatment Panel III). JAMA 2001; 285: 2486-2497.

14 World Health Organization-International Society of Hypertension Guidelines for the management of hypertension. J Hypertens 1999; 17: 151-183.

15 Mancia G, De Backer G, Dominiczak A, Cifkova R, Fagard R, Germano G, Grassi G, Heagerty AM, Kjeldsen SE, Laurent S, Narkiewicz K, Ruilope L, Rynkiewicz A,
Schmieder RE, Boudier HA, Zanchetti A, Vahanian A, Camm J, De Caterina R, Dean V, Dickstein K, Filippatos G, Funck-Brentano C, Hellemans I, Kristensen SD, McGregor K, Sechtem U, Silber S, Tendera M, Widimsky P, Zamorano JL, Erdine S, Kiowski W, Agabiti-Rosei E, Ambrosioni E, Lindholm LH, Viigimaa M, Adamopoulos S, Agabiti-Rosei E, Ambrosioni E, Bertomeu V, Clement D, Erdine S, Farsang C, Gaita D, Lip G, Mallion JM, Manolis AJ, Nilsson PM, O'Brien E, Ponikowski P, Redon J, Ruschitzka F, Tamargo J, van Zwieten P, Waeber B, Williams B, Management of Arterial Hypertension of the European Society of Hypertension; European Society of Cardiology Guidelines for the Management of Arterial Hypertension. The Task Force for the Management of Arterial Hypertension of the European Society of Hypertension (ESH) and of the European Society of Cardiology (ESC). J Hypertens 2007; 25: 1105-1187.

16 The Seventh Report of the Joint National Committee on Prevention, Detection, Evaluation, and Treatment of High Blood Pressure: the JNC 7 report. JAMA 2003; 289: 2560-2572.

17 Artigao LM, Llavador JJ, Puras A, López Abril J, Rubio MM, Torres C, Vidal A, Sanchis C, Divisón JA, Naharro F, Caldevilla D, Fuentes G. Evaluation and validation of Omron Hem $705 \mathrm{CP}$ and Hem 706/711 monitors for self-measurement of blood pressure. Aten Primaria 2000; 25: 96-102.

18 Foster C, McKinlay S, Cruickshank JM, Coats A. Accuracy of the Omron HEM 706 portable monitor for home measurement of blood pressure. J Hum Hypertens 1994; 8: 661-664.

19 O'Brien E, Mee F, Atkins N, O'Malley K. Short report: accuracy of the Dinamap portable monitor, model 8100 determined by the British Hypertension Society protocol. J Hypertens 1993; 11: 761-763.

20 Association for the Advancement of Medical Instrumentation (AAMI). American National Standard for Electronic or automated Sphygmomanometer. AAMI: Arlington, VA, 1987.

21 Domingo A, Marcos J. Proposal of an indicator of 'social class' based on the occupation. Gac Sanit 1989; 3: 320-326.

22 Mahan LK, Arlin MT eds) Nutrición y Dietoterapia. Interamericana: McGraw-Hill, Mexico DF, 1995, pp 17-28.

23 Gonzalez JR, Armengol L, Sole X, Guinó E, Mercader JM, Estivill X, Moreno V. SNPassoc: an $\mathrm{R}$ package to perform whole genome association studies. Bioinformatics 2007; 23: 644-645.

24 Ding EL, Smit LA, Hu FB. The metabolic syndrome as a cluster of risk factors: is the whole greater than the sum of its parts? Arch Intern Med 2010; 170: 484-485.

25 Martinez MA, Puig JG, Mora M, Aragon R, O'Dogherty P, Anton JL, Sánchez-Villares T, Rubio JM, Rosado J, Torres R, Marcos J, Pallardo LF, Banegas JR, MAPA (Monitorizaci ó n Ambulatoria de la Presi ó n Arterial) Working Group. Metabolic syndrome: prevalence, associated factors, and C-reactive protein: the MADRIC study. Metabolism 2008; 57 : 1232-1240.

26 Buckland G, Salas-Salvado J, Roure E, Bullo M, Serra-Majem L. Sociodemographic risk factors associated with metabolic syndrome in a mediterranean population. Public Health Nutr 2008; 11: 1372-1378.

27 Alonso-Moreno FJ, Llisterri Caro JL, Rodriguez-Roca GC, Ferreiro Madueno M, Gonzalez-Segura Alsina D, Divison Garrote JA, Banegas JR, Barrios Alonso V, Lou Arnal S, Sánchez Ruiz T, Santos Rodríguez JA, Durá Belinchón R, Grupo de Trabajo de Hipertensi ó n Arterial de la Sociedad Espa ñ ola de Médicos de Atenci ó n Primaria (Grupo HTA/SEMERGEN); Estudio PRESCAP 2006. Primary care physicians behaviour on hypertensive patients with poor blood pressure control. The PRESCAP 2006 study. Rev Clin Esp 2008; 208: 393-399.

28 Márquez-Contreras E, Martel-Claros N, Gil-Guillén V, Martín-De Pablos JL, De La Figuera Von Wichman M, Casado-Martínez JJ, Espinosa-García J. Control of therapeutic inertia in the treatment of arterial hypertension by using different strategies. Aten Primaria 2009; 41: 315-323.

29 Whaley-Connell A, Pavey BS, Chaudhary K, Saab G, Sowers JR. Renin-angiotensinaldosterone system intervention in the cardiometabolic syndrome and cardio-renal protection. Ther Adv Cardiovasc Dis 2007; 1: 27-35.

30 Konoshita T, Kato N, Fuchs S, Mizuno S, Aoyama C, Motomura M, Makino Y, Wakahara S, Inoki I, Miyamori I, Pinet F, Genomic Disease Outcome Consortium (G-DOC) Study Investigators. Genetic variant of the renin-angiotensin system and diabetes influences blood pressure response to angiotensin receptor blockers. Diabetes Care 2009; 32: 1485-1490. 